Introduction In 2015, the estimated HIV prevalence in Brazil was $0.4 \%$. This figure has been stable in recent years, but it can mask disparities among regions. In this study we present the spatial distribution of the variation in HIV incidence rates (IR) in Brazilian municipalities from 2009-14 and assess the existence of spatial clustering of increase or decrease in these IR.

Methods We used the AIDS reporting system(Sinan) and programmatic data on Viral Load(VL) exams and ARVs. The diagnosis date used was the earliest among $1^{\text {st }}$ detectable VL, $1^{\text {st }}$ ARV dispensation or diagnosis date in Sinan. Annual IR were generated by municipality. To smooth the IR, we used 3 year averages and applied the local empirical Bayesian method. To assess IR time trends, we calculated the percent IR variations in the period. For spatial statistical analysis, a simple adjacency matrix was generated, and Global and Local Moran's I autocorrelation tests were applied.

Results The Global Moran's I for the IR variation was 0.42 $(\mathrm{p}<0.001)$ which points to spatial clustering. We generated 2 maps, one for the percent IR variation and another to represent the statistically significant high-high and low-low clusters. In the $1^{\text {st }}$, we observed that most municipalities in the North $(\mathrm{N})$ and Center-West $(\mathrm{CW})$ present increases in the period; in the Southeast(SE), the state of Sao Paulo(SP) reveals the most relevant decreases in the country; the Northeast(NE), South(S) and some areas of the SE show mixed patterns. The $2^{\text {nd }}$ map makes regional disparities even clearer. There are big clusters of increasing IR in most states of the $\mathrm{N}$, and smaller ones in areas of the CW and NE. Several clusters of declining IR are seen in SP, Minas Gerais(SE), Santa Catarina, Rio Grande do $\mathrm{Sul}(\mathrm{S})$, and areas of the NE.

Conclusion Spatial dependency in HIV IR variations in Brazil was evidenced. The methods used in this study have proved useful in monitoring spatiotemporal trends, pointing out important regional differences. Similar analysis can be performed at state and city levels, contributing to improved diagnoses of local epidemics.

\section{P3.150 IMPORTANCE OF REGIONAL OFFICES ON THE IST PROTOCOL OF THE MINISTRY OF HEALTH FOR MULTIPLICATOR FORMATION AND CONTROL OF THESE INFECTIONS IN BRAZIL}

Maria Vitória Ramos Gonçalves, JP Toledo, FLS Freitas, AS Benzaken.

\subsection{6/sextrans-2017-053264.385}

Departamento de Vigilância, Prevenção e Controle das Infecções Sexualmente Transmissíveis, do HIV/AIDS Brasilia - DF - Brazil Introduction According to the World Health Organisation, more than a million STIs are acquiredevery day. In 2012, an estimated 357 million new cases of curable STIs (gonorrhoea, chlamydia, syphilis and trichomoniasis). The care for clients with STIs is mostly performed in the Primary Care service $(\mathrm{AB})$, in order to stop the signs and symptoms, preventing these infections from becoming complicated, interrupting the transmission chain as effectively and immediately as possible. After ten years of publication of theManual on the Control of Sexually Transmitted Diseases, the Department of STIs, HIV/ AIDS and Viral Hepatitis (DIAHV) of the Ministry of Health (MS) innovates and launches a Clinical Protocol and Therapeutic Guidelines for Integral Care for Persons with Sexually Transmitted Infections Transmissible - PCDT/IST, considering the need to hold regional workshops for multipliers in the territories. To align and integrate prevention, care, treatment and surveillance actions in the health services, strengthen the work of health professionals in STI/HIV/Aids, according to PCDT/ IST; identify the professional's abilities to perform the appropriate management of care for people with STIs; to discuss the flowcharts and protocols used in the management of STIs. Methods Using the methodology of the problematization, which proposes the construction of knowledge, based on the reality of the participants and favours the joint reflection and the exchange of experiences. For the realisation of the workshops in the regions, the DIAHV counted on partnership of the states.

Results More than 400 professionals (doctors and nurses) from specialised services and $A B$ were trained in four regions of the country.

Conclusion The realisation of these workshops and the interaction of the DIAHV/MS technicians with care professionals contributed to standardise the management of STIs and to qualify health care for people with STIs. These professionals were certified to multiply the workshop to their peers and send reports of actions to the state.

\section{P3.151 THE ROUTES OF THE AIDS AND HIV INFECTION EPIDEMIC IN THE STATE OF SÃO PAULO, BRAZIL, BETWEEN 2000 AND 2015}

Mariza Vono Tancredi, CSB Domingues, A Tayra, MA Silva, MC Gianna. Centro de Referência e Treinamento DST/AIDS-SP, São Paulo - SP, Brazil

\subsection{6/sextrans-2017-053264.386}

Introduction This study analysed the HIV/AIDS epidemic trends in the state of Sao Paulo, in adults, during the period of 2000 to 2015 .

Methods Trends study performed by polynomial regression model, with AIDS and HIV positive cases notification comparing by age group and exposure categories. The dependent variable were the annual number of cases, in each of the studied categories, and the independent variable was the time, (calendar years), concerning the study period. The goodness of fit via $\mathrm{r}^{2}$ and $\mathrm{p}<0.05$ were used to determine which models and data were most appropriate.

Results It was analysed 142,015 AIDS cases and 70761 cases of HIV. The AIDS cases trends declined in the entire period, with speed $=243$ cases $/$ year $(p=0.001)$, but the HIV + cases trends increased with speed $=325$ cases $/$ year $(p=0.003)$. From 2000 to 2007 AIDS decreased 30 cases/year $(p=0.024)$ in the man who have sex with man (MSM) category, and only the $30-39$ years group falled 26 cases/year $(p=0.003)$. From 2007 to 2012 there was a growth of MSM with speed=116 cases/ year $(p<0.001)$ and the $20-24$ years group showed the fastest growth $=38$ cases/year $(p=0.001)$. From 2012 to 2015 , the MSM decreased 118 cases/year $(p=0.043)$ and the 30-39 years group showed a greater fall rate $=43$ cases $/$ year $(\mathrm{p}=0.039)$. Among the HIV infection cases in the period 2000 to 2003, the trend was increasing among MSM with speed $=201$ cases $/$ year $(p<0.001)$ with the group 30-39 years growing faster $=79$ cases/year $(p=0.006)$. Between 2003 and 2007 MSM increased 25 cases/year $(p=0.048)$ and the age group 40-49 presented the highest velocity $=8$ cases/year $(p=0.006)$. Between 2007 and 2015 the trend was increasing among MSM with speed $=337$ cases $/$ year $(p=0.001)$ and the 